Journal Plus Education, ISSN: 1842-077X, E-ISSN (online) 2068-1151 Vol XIX (2018), No. 1. pp. 207-219

\title{
PARTICULARITIES OF THE DEVELOPMENT OF THINKING AND LANGUAGE IN PRE-SCHOOLERS
}

\author{
Tiberiu DUGHI, PhD, \\ Universitatea „Aurel Vlaicu” Arad \\ Carmen ROPOTA, master student, \\ Universitatea „Aurel Vlaicu” Arad \\ tibi dughi@yahoo.com
}

\begin{abstract}
The article highlights particularities related to the development of thinking and language in preschool children and the importance of these two aspects in the formation of the child's personality and in the formation of the necessary skills for schooling. In order to get the arguments and formulations of hypotheses, we used the Goodenough Test and the Screening of Cognitive Skills, the form for educators, SCC 4-5 years, Screening of School Pre-requisites, form for educators, SPS 4-5 years. These samples were applied to a sample of 22 subjects, girls and boys aged 4 to 5 years.
\end{abstract}

Key words: thinking; language; children; evaluation; skills for schooling;

\section{Introduction}

The evolution of the personality of each individual depends on the basis of development. These bases are laid down in the preschool age, which is considered a quiet period, between a strong development and the increase of the first years of life and the school age. Therefore, it is a suitable age for the development of the main psychic processes (memory, thinking, language and emotional affective manifestations).

Thinking plays an important role in the human psychic system, being defined as a succession of operations that will lead to the disclosure of important life hypotheses and to solving certain problems. During preschool, brain activity is intense, at this age child ask a lot of questions about any aspect of reality. Through language, preschool develops autonomy, also gives him the ability to move easily into the environment and to interact with people around him. Therefore, the ability to communicate with those around him helps the child to express his thoughts, ideas, impressions so that he can understand and form a basis for his school and social activity. 


\section{Theoretical foundation}

Pre-schooling, the development period of 3-6 / 7 years of age, brings a series of changes in both mental and relational development plans. During pre-school, brain activity is intense, children ask questions about any aspect. Therefore, it is advisable to respond calmly and gently to all questions, because at this time the children accumulate important information, even more than during schooling.

, Thinking of the preschool is intuitive and preoperative. It is a preconception and quasi-conceptual thinking, which means that it operates with a series of constructs that are neither individualized notions or general notions. The concrete thinking of the child differs very little from his real impressions. The child is capable of symbolic thinking with the use of words.' (Schwartz, G., Kelemen, G., 2006, p. 94)

Preconception and intuitive thinking is an egocentric thinking, failing to distinguish between objective and personal reality, generating egocentrism, preschooler being the center of the universe, believing that everything is "spinning" around him.

Around the age of 3 , the child is at the start of the preoperative stage because the child does not understand the certain rules or operations yet. Acording to Piaget, the child at this stage has not acquired the conservation of invariants such as quantity, weight or volume. (Moldovan, Ignat, BălașTimar,2011)

The preschool begins to speak, to use mental symbols, thus becoming able to use thoughts and ideas in order to better understand what surrounds him. At this age, he has the opportunity to play certain roles in symbolic games such as "mother", "father", "doctor", etc. These games aim to develop imaginary game, as words are meant to replace concrete situations. At the pre-school age, the child can only manipulate the objects in front of him, he cannot imagine and cannot work with ideas and things that are abstract.

Through logical classifications, children understand the relationship between events and unite experiences. "The ability to achieve classifications increases during pre-school years, but remains fragile and suffers a decrease when the situation becomes larger. When children collect stamps, they organize them according to different criteria: stamps come from different countries, from different years, they are from different domains. Then, preschoolers are not able to achieve complex object classifications, using few attributes of objects. " (Golu, F., 2015, p. 103)

Another aspect of thinking at this preoperative stage is causality. In this period of early childhood, children are not capable to understand the cause and effect, they correlate certain phenomena even though there is no causal relationship between them. This is the "why" period that every child goes through at this age. The answers we give to the child must have an 
educational and informative role. They will often be the starting point for other questions that the child will continue to ask.

„Bruner (1987) suggests that the reason a child learns to speak is his desire to communicate with the person who takes care of him. (Muntean, A., 2006)

Language acquisition involves a number of specific skills and abilities. As with motor development, age, maturation process is important, because when they are raised in typical conditions, children acquire language after a program they have in the nervous system as part of their genetic endowment." (Schaffer, 2007, p.286)

Language development for preschoolers is strongly influenced by the environment in which child lives, how much their parents speak with him, how much he is encouraged to use language in communication. That is why at this age there are differences between children (if is not properly stimulated the child will speak later). Acquiring verbal structures is done by imitating parents, and then by respecting the models proposed by the kindergarten. Kindergarten provides pre-school students with knowledge and helps them to form skills to properly flex nouns by gender, number and case, and verbs by person, number and time.

When entering the institutionalized kindergarten, pre-school children have different communication experiences, either with other children or with adults, and this is closely related to the environment they come from. Therefore, at this stage, adults must focus on the content of language with the child, they should teach the child the names of objects, formulate and pronounce correctly words and sentences.

At the same time, several types of language are used in this age. The "egocentric language" is described by Piaget as a "conversation" with its own person, meaning a certain exercise for the future social language. This discussion is aimed both at pronouncing the words correctly and in supporting the various activities they perform individually. The child may be near another person, an adult or a child, but he speaks without the intention of communicating to him, without wanting to receive what he says and understand. It is therefore an intermediate form between the actual and the external language itself. (Tinca Cretu, 2016)

Another type of language used by preschoolers is the collective monologue that is predominantly encountered in groups of children where everyone seems to speak only to him using his own language without being bothered by what his colleagues say. The socialized language is related to the needs of other people around, aiming to establish contacts and relationships.

At present, pre-school children enter in the preparatory class with a special training. Kindergarten, being the first link of the education system, will need to know how children's intellect works, to identify the areas in 
which they are inclined to predict each child. The child who is fit to be taught has some skills: to observe, to analyze, to listen to certain requirements, to answer questions, to formulate, to complete or correct colleagues' answers, these skills being in the teacher's attention throughout the kindergarten.

The observation activity carried out in the kindergarten involves the active and systematic perception of objects or phenomena of the environment, being a source of impressions that pre-school children accumulate and exploit throughout their lives. Through this activity, the sensory basis of the knowledge process will develop, stimulate intellectual processes, ensure the understanding of simple relationships between objects and phenomena of nature. The most important part of the observation is the perception of the material, its analysis with the help of sense organs, directly involved in thinking operations such as analysis and synthesis. This method is dominated by communication and assimilation of knowledge, being necessary for adaptation at school entrance.

At the same time, stories and retellings have a great importance in cognitive and language development because at the entrance to school many students do not know how to express their opinion, their vocabulary being made up of few words and expressions. With the help of stories, children learn new phrases, which once known, come into their vocabulary. "Besides giving the child the opportunity to learn to understand the thoughts and feelings of the people, he acquaints the child with the structure of the language, with the richness of the grammatical forms, with the expressivity of the tongue" (Tătaru, L., Glava, A., Chiş, O., 2014, p. 209) Also, through the stories, pre-school children learn to distinguish the good from evil, they even find models of positive heroes, influencing the formation of children's personality and their behavior and attitude in certain situations of life .

The development of language implies the assimilation of a lexical background and the meaning of words, grammatical structures. By organizing of didactic games such as "I say one, you say a lot", preschooler will have the task of correctly using the singular and plural number of nouns in communication, a didactic game such a,, Multiple Meaning Words" where the child will identify the different meanings of the words with the help of images, another example of a game is" Who You Send Letters ", a game which the child will learn to use the dative in the expression correctly.

Learning graphs plays an important role in preparation for school because it teaches pre-school children to write certain graphic signs that will later become letters. So, at the end of kindergarten, the child will know certain letters of the alphabet, he / she will be able to write his own name, which will help him / her in the development of the written language. 


\section{Objectives of the study}

O1. Assessment of cognitive skills of the 4-5-year-old child: attention, memory, categorization, language, problem planning and problem solving, as observed by the educator.

$\mathrm{O} 2$. Assessment of pre-requisites for schooling: prerequisites for writing and reading, mathematical prerequisites, as well as knowledge about cognitive functioning, as observed by the educator.

O3. The evaluation of intelligence, respectively the degree of intellectual maturation of children of 4-5 years, expressed by drawing.

\section{The assumptions of the study}

Hypothesis 1: There is a statistically significant (positive) direct correlation between the child's cognitive skills of 4-5 years and the prerequisites of schooling.

Hypothesis 2: There is a statistically significant (positive) direct correlation between the level of intelligence (intellectual maturation degree) and the cognitive skills of the child for $4-5$ years.

Hypothesis 3: There is a statistically significant (positive) direct correlation between the level of intelligence (degree of intellectual maturation) and the pre-requisites of schooling.

\section{Sample}

The working tools focused on a sample of 22 pre-school children aged between 4 and 5 years, from Kindergarten P.P. Number 14, Arad. The scales of cognitive skills screening and pre-requisites for schooling were completed by the teacher for each child in the sample, according to the methodological evaluation procedures. The Goodenough test was applied to children in the sample. The study was conducted in March 2017.

The age group of 4-5 years has been chosen because at this stage both thinking and language are in the process of being developed, and any pronunciation errors or problems in the cognitive sphere can be remedied. All children in the sample are part of the same group. The group follows a traditional curriculum and is part of mass education. Subjects come from normal families that do not have dysfunctions.

Regarding the distribution of subjects investigated by gender / sex, the preschool group consists of 12 boys and 10 girls 
Table 1: Distribution of the investigated sample by sex / gender

\begin{tabular}{|ll|l|l|l|l|}
\hline & Sex & Frequency & Percent & Valid Percent & $\begin{array}{l}\text { Cumulative } \\
\text { Percent }\end{array}$ \\
\hline Valid & Boys & 12 & 54,5 & 54,5 & 54,5 \\
& Girls & 10 & 45,5 & 45,5 & 100,0 \\
& Total & 22 & 100,0 & 100,0 & \\
\hline
\end{tabular}

\section{Methods and tests used in research}

During the research, I wanted to give arguments in favor of the hypotheses formulated, that is why I used the Goodenough Test, the Cognitive Skills Screening for 4-5 years, the form for educators and the Screening of the pre-requisites of the schooling.

The Goodenough test - the test of the little man, highlights at least three aspects that can be considered separately or correlatively: cognitive development (the most widely accepted and most validated psychometric), dimension related to the personality plan and one that shows an exceptional ability or talent for drawing (used in selection but also in vocational training). We have applied this test to determine the degree of cognitive development of middle-class preschoolers.

This test was applied individually to each preschool. Each subject received a piece of paper, a box of seven colored pencils: blue, green, red, yellow, purple, brown, black, as well as a black pencil, an eraser. The educator told to children that they would draw a little man on that sheet of paper as beautiful as they can and if they want they can paint their draw. The subject will be left to draw as he wishes without support and without criticism, appreciation or suggestion

Preschoolers who are undecided can be encouraged with formulas such as: "You are doing very well, continue." If the child asks how to draw the teacher answer: "Do what you want, how you want to do."

The subject will be left to choose on which part of the sheet he draws, what dimensions will have his draw, the character of the drawn character, theme, the colors used or to refuse to paint his draw, each choice that child makes has a meaning.

Cognitive Skills Screening, Form for Educators-SCC-E 4-5 (by COGNITROM) evaluates cognitive skills from the perspective of several features: attention, memory, categorization, problem-solving and problemsolving (comprehension and expression). To evaluate cognitive skills, we used the 4-5-year scale (from 4 years to one day and up to 5 years), the variants for educators. 
Each item has an answer on a 5-point scale, where 1 signifies almost never, and 5 means almost always. The educator will circle only one answer to each item. If the person completing that questionnaire did not notice the behavior described in those statements, she would have to approximate a response that is closest to the child's behavior.

The screening of cognitive skills, the form for educators - SCC-E 4-5, contains 21 items describing a series of behaviors that preschoolers 4-5 years of age should do. These behaviors were observed by the educator during the group activities. Items have been distributed in several sizes:

- Items 1 and 2 evaluate the attention;

- Items: 3,4 and 5 belong to the memory dimension;

- Items 6, 7 and 8 belong to the rating dimension: solving;

- Items 9,10,11,12 belong to the dimension planning and problem

- Items 13, 14, 15, 16 evaluate comprehension, and items 17, 18, 19, 20, 21 refer to expression, also, they belong to the language dimension.

At the age of 4-5 years, an improvement in focus may be seen. Children can concentrate in an individual activity for about 10-15 minutes, and in group tasks about 20 minutes.

With regard to memory, children can tell a story that has been heard, especially because language skills are better developed.

Also, the categorization becomes more extensive. Children can group different objects according to two criteria at the same time: by the form and color or by the size and utility. At this age, the preschool can identify, using images, sequences that refer to four actions and can place them in the chronological order of deployment. At the same time, the child can solve a problem without the adult's intervention, such as the loss of a favorite toy.

From the point of view of comprehension and language expression, they take increasingly complex forms, starting from the identification of simple rhymes and ending with the rendering of the main ideas of stories and details from the stories.

Another questionnaire applied to preschoolers is Screening of prerequisites for schooling, version for educators - SPS 4-5 - Cognitrom. Prerequisites for schooling are specific academic or cognitive abilities. This questionnaire contains 14 relevant items for the age of 4-5 years.

Scale items are statements describing those defining behaviors that a child needs to acquire according to his cognitive development. The educator will read the 14 items and will try the YES if the preschool has the experience and NOT if he / she does not show the skills. A single answer will be marked on each affirmation, and if the educator is not sure of the statement, he will choose the nearest answer to what he thinks. 
Items are distributed according to three dimensions:

- Items 1, 2, 9, 10, 11, 12, 13 comprise the field of written and read pre-requisites;

- Items 3, 4, 5, 6 belong to the mathematical prerequisite;

- Items 7, 8, 14 encompass knowledge about cognitive functioning.

In the case of educators, the number of items for reading and writing is higher because they have more information about these aspects. For the ability to write and read, it is very important to trace lines in zigzag, color the inside of a circle, copy certain geometric figures following a given pattern. Regarding mathematical prerequisites, it is essential that the preschool knows how to count up to five and to locate the number in the range of objects.

Concerning knowledge about cognitive functioning, the scale includes items starting from "mind theory", meaning that the child understands that two people can have different views on the same situation. This subscale also includes items describing the awareness of certain limits of capacity, the child realizes that it cannot solve a task. Since the child can distinguish between reality and fiction at the age of 4-5 years, thus exceeding the stage of thinking magical, realizing that the characters in the stories do not actually exist, the assessment of this aspect is important for the cognitive development of preschoolers.

\section{Data analysis.}

Hypothesis 1: There is a statistically significant (positive) direct correlation between the child's cognitive skills of 4-5 years and the prerequisites of schooling.

To test this hypothesis, we used the Pearson correlation coefficient.

Table 2 on the correlation between cognitive skills and pre-requisites for schooling

\section{Correlations}

\begin{tabular}{|ll|l|l|}
\hline & & $\begin{array}{l}\text { Cognitive } \\
\text { skills }\end{array}$ & $\begin{array}{l}\text { Prerequisites } \\
\text { for schooling }\end{array}$ \\
\hline Cognitive skills & Pearson Correlation & 1 &, $598^{* *}$ \\
& Sig. (2-tailed) & &, 003 \\
$\mathrm{~N}$ & 22 & 22 \\
\hline $\begin{array}{l}\text { Prerequisites } \\
\text { schooling }\end{array}$ & for Pearson Correlation &, $598^{* *}$ & 1 \\
& Sig. (2-tailed) &, 003 & \\
& $\mathrm{~N}$ & 22 & 22 \\
\hline
\end{tabular}


Journal Plus Education, ISSN: 1842-077X, E-ISSN (online) 2068-1151 Vol XIX (2018), No. 1. pp. 207-219

\section{Correlations}

\begin{tabular}{|lc|l|l|}
\hline & & $\begin{array}{l}\text { Cognitive } \\
\text { skills }\end{array}$ & $\begin{array}{l}\text { Prerequisites } \\
\text { for schooling }\end{array}$ \\
\hline Cognitive skills & Pearson Correlation & 1 &, $598^{* *}$ \\
& Sig. (2-tailed) & &, 003 \\
& $\mathrm{~N}$ & 22 & 22 \\
\hline $\begin{array}{l}\text { Prerequisites } \\
\text { schooling }\end{array}$ & for Pearson Correlation &, $598^{* *}$ & 1 \\
& Sig. (2-tailed) &, 003 & \\
& $\mathrm{~N}$ & 22 & 22 \\
\hline
\end{tabular}

${ }^{* *}$. Correlation is significant at the 0.01 level (2-tailed).

According to the table, a correlation coefficient $r=0.598$ significantly was obtained at a threshold of $\mathrm{p}<0.01$, between the cognitive competences and the pre-requisites of the schooling. Thus, hypothesis 1 is confirmed, there is a statistically significant (positive) correlation between the cognitive skills of the 4-5-year-old child and the pre-requisites of schooling, between the two variables being a positive, directly proportional correlation. That is, an increase or decrease in the values of the first variable (Cognitive Skills) corresponds to an increase or decrease of the second variable (Prerequisites of Schooling). The higher or lower the level of cognitive competence development, the higher / lower the level of acquisition of schooling prerequisites.

The value of the Pearson coefficient is 0.598 , which indicates the existence of a medium intensity correlation. The significance threshold is 0.003 , that is, less than 0.01 , indicating that the test is significant.

If a child is able to focus on an activity for a certain period of time, he knows the figures by their form, but also associates the figure with the quantity, if he easily memorizes poems and songs, if he knows how to divide objects into groups according to color, thickness, size, if he understands and can tell with his words a heard story and describe some details of that story, if he manages to draw some geometric figures without help or if he colors the inside of a figure without exceeding the contour, then the child presents and a significant development of pre-requisites for schooling.

Hypothesis 2: There is a statistically significant (positive) direct correlation between the level of intelligence (intellectual maturation degree) and the cognitive skills of the child for 4-5 years.

To test this hypothesis, we used the Pearson correlation coefficient. 
Table 3: The correlation between the level of intelligence (degree of intellectual maturity) and cognitive skills

\section{Correlations}

\begin{tabular}{|ll|l|l|}
\hline & & $\begin{array}{l}\text { Intelligence } \\
\text { degree of } \\
\text { intellectual } \\
\text { maturity }\end{array}$ \\
\hline Cognitive skills & $\begin{array}{l}\text { Pearson } \\
\text { Skills }\end{array}$ & 1 &, $702^{* *}$ \\
& $\begin{array}{l}\text { Correlation } \\
\text { Sig. (2-tailed) } \\
\text { N }\end{array}$ & 22 &, 000 \\
Intelligence / degree of Pearson &, $702^{* *}$ & 22 \\
intellectual maturity $\quad$ Correlation & & 1 \\
& Sig. (2-tailed) &, 000 & 22 \\
N & 22 & \\
\hline
\end{tabular}

**. Correlation is significant at the 0.01 level (2-tailed).

According to the table, a correlation coefficient $r=0.702$ was obtained at a threshold $\mathrm{p}<0.01$, between the level of intelligence (intellectual maturation degree) and cognitive skills. Thus I. 2 is confirmed, between the two variables there is a positive correlation, directly proportional. That is, an increase or a decrease in the values of the first variable (Intelligence / Degree of Intellectual Maturity) corresponds to an increase or decrease of the second variable (cognitive competences). The higher / lower the level of intelligence / intellectual maturation, the higher / lower the level of cognitive competence development.

The value of the Pearson coefficient is 0.702 , indicating a correlation of medium to good intensity. The significance threshold is 0.000 , that is, less than 0.01 , which indicates that the test is significant.

If a child has a good intellectual maturity (good degree of intellectual maturation), then that child is able to focus attention, also he can easily memorize poems, he knows how to share objects in groups according to color, thickness, size, understanding and he can tell a story with his own words.

Hypothesis 3: There is a statistically significant (positive) correlation between the level of intelligence (the degree of intellectual maturation) and the pre-requisites of schooling. To test this hypothesis, we used the Pearson correlation coefficient. 
Table 4: Correlation between the level of intelligence (degree of intellectual maturity) and

prerequisites for schooling

\section{Correlations}

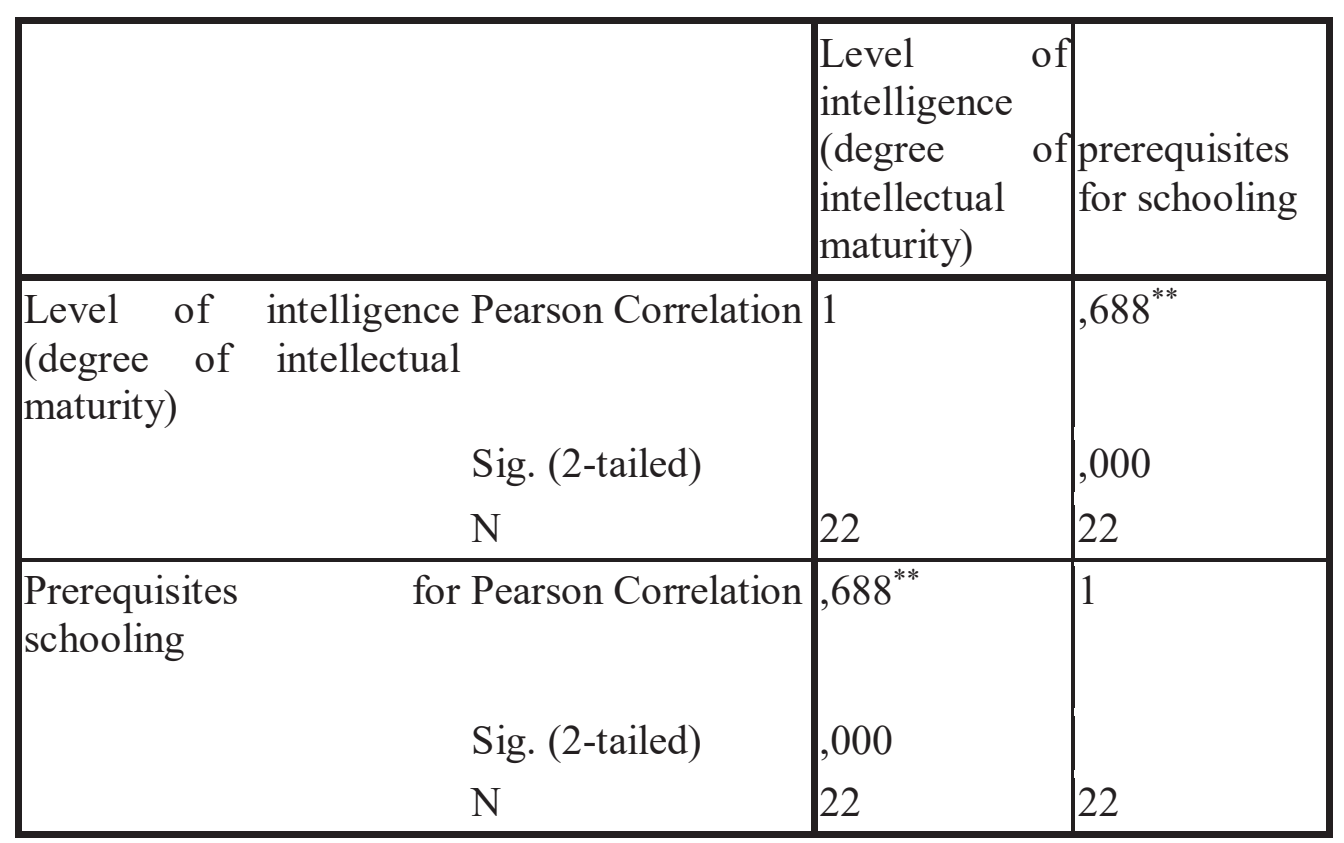

**. Correlation is significant at the 0.01 level (2-tailed).

According to the table, a correlation coefficient $r=0.688$ significant was obtained at a threshold $\mathrm{p}<0.01$, between the level of intelligence (intellectual maturation degree) and the cognitive skills. Thus hypothesis 3 is confirmed, between the two variables being a positive correlation, directly proportional. That is, an increase or decrease in the values of the first variable (Intelligence / Degree of Intellectual Maturity) corresponds to an increase or decrease of the second variable (the prerequisites of the schooling).

The higher / lower the level of intelligence / intellectual maturation, the higher / lower the level of acquisition of pre-requisites for schooling.

The value of the Pearson coefficient is 0.688 , indicating the existence of a medium intensity correlation. The significance threshold is 0.000 , that is, less than 0.01 , which indicates that the test is significant.

If a child has a good intellectual (good degree of intellectual maturity), then the child also has a significant development of the pre-requisites of schooling, he will be able to adapt to the requirements of schooling, he can 
perform simple assembly and lowering operations, also he can write or read by doing these actions consciously.

\section{Conclusions}

The data obtained from the research confirmed the first hypothesis from which we started, namely that there is a direct correlation between the child's cognitive competences of 4-5 years and the pre-requisites of schooling. Therefore, the higher or lower the level of cognitive competence development, the higher / lower the level of acquisition of schooling prerequisites.

When a child can focus on an activity, he knows the figures by their form, but also associates the figure with the quantity, if he easily memorizes poems and songs, if he can share objects in groups according to color, thickness, size, if he understands and can tell a story with his words, if he manages to draw some geometric figures without help, or if he colors the inside of a figure without exceeding the contour, then the child also presents a significant development of the prerequisites of schooling.

At the same time, the second hypothesis, which expresses the fact that there is a direct positive correlation between the intelligence level and the cognitive skills of the child, was confirmed because a correlation coefficient $\mathrm{r}=0.702$ significant was obtained at a threshold $\mathrm{p}<0.01$, between the level of intelligence (degree of intellectual maturation) and cognitive skills. If a child has a good intellectual maturity (good degree of intellectual maturation), then that child is able to focus attention, easily memorize poems, knows how to share objects in groups according to color, thickness, size, understands and can tell with his words a heard story.

The third hypothesis regarding the existence of a direct, statistically significant correlation between the level of intelligence and the pre-requisites of schooling is confirmed. The value of the Pearson coefficient is 0.688 , indicating the existence of a medium intensity correlation. The significance threshold is 0.000 , that is, less than 0.01 , which indicates that the test is significant. If a child has a good intellectual (good intellectual maturity) coefficient, then that child also has a significant development of school prerequisites.

One of the limits of the research would be the small size of the sample (22 subjects) with repercussions on representativeness. Another limit of research would be the lack of comparison with other groups (preschoolers from other groups, other kindergartens, other educational alternatives, other forms of funding). Investigating intelligence through a single projective test, the Goodenough test, is another limit of this research because applying other intelligence tests on the same sample will bring more accuracy. 
In the future, this research could continue using procedures to configure a representative sample of subjects for more significant accuracy. Also, we could investigate the thinking, language and prerequisites of schooling through other tools (other questionnaires, scales, tests) so that the results obtained can be compared to each other.

\section{References:}

Crețu, T., (2016), Psihologia vârstelor, Polirom, Iași

Golu, F., (2015), Manual de psihologia dezvoltării: o abordare psihodinamică, Polirom, Iași

Moldovan, O., S. Ignat, S., Bălaş-Timar, D., (2011), Fundamentele Psihologiei I, Editura Universităţii „Aurel Vlaicu” Arad.

Muntean, A., (2006), Psihologia dezvoltării umane, Editura Polirom, Iaşi

Schwartz, Gh., Kelemen, G., (2006) Psihologia copilului, Editura Universităţii „Aurel Vlaicu” Arad.

Shaffer, R., (2007), Introducere în psihologia copilului, Editura ASCR, Cluj Napoca

Tătaru, L., Glava, A., Chiş, O., (2014) Piramida Cunoaşterii-repere metodice în aplicarea curriculumului preșcolar, Editura Diamant, Pitești. 